\title{
The Orbits of Meteorites from Natural Thermoluminescence
}

\author{
P. H. Benolt and D. W. G. Sears \\ Cosmochemistry Group, Department of Chemistry and Biochemistry. University of Arkansas. Favetteville. Arkansas 72701 \\ E·mail: cosmo@uafsysb.uark.edu
}

Received December 15, 1995: revised July 29, 1996

The natural thermoluminescence (TL) of meteorites reflects their irradiation and thermal histories. Virtually all ordinary chondrites have been irradiated long enough to have reach saturation natural TL levels, and thus natural TL levels in these meteorites are determined largely by thermal history. The primary heat source for most meteorites is the Sun, and thus natural TL levels are determined primarily by the closest approach to the Sun, i.e., perihelion. By converting natural TL levels to perihelia, using an assumed albedo typical of meteoroid bodies, it is found that most ordinary chondrites had perihelia of 0.85 to $1.0 \mathrm{AU}$ prior to reaching Earth. This range is similar to that calculated from meteor and fireball observations. All common classes of ordinary chondrites exhibit similar perihelia distributions; however, $\mathrm{H}$ and $\mathrm{LL}$ chondrites that fell in the local morning differ in their natural TL distribution from those that fell in the local afternoon and evening. This is consistent with earlier suggestions that time of fall reflects orbital distribution. The data also suggest that the orbits of some of the $H$ chondrites cluster and may have come from a debris "stream" of meteoroids. If meteorites can exist in "orbital groups," significant changes in the types and number of meteorites reaching Earth could occur on the $<10^{5}$-year time scale. 1997 Academic Press

\section{INTRODUCTION}

Most meteorites are thought to be samples of asteroids (Wetherill 1985); however. it is unlikely that meteorites are random representative samples of their parent bodies. Their passage to Earth depends on their original orbit and the stochastic nature of the collisional events that ejected them into space (Wetherill 1985. Greenberg and Nolan 1989. Binzel et al. 1993). It has been proposed that some fraction of the meteorite flux derives from coherent orbital "streams" of meteoroids (Hallidav 1987, Dodd et al. 1993). This idea is indirectly supported by orbital clustering of meteor-producing bodies and temporal clusters of meteoroid impacts on the Moon (Olsson-Steel 1988, Halliday $e t$ al. 1990. Drummond 1991. Oberst and Nakamura 1991). There is. however. little evidence that, most meteorites were members of meteoroid streams at the time of their fall (Wetherill 1985).
The possible existence of meteoroid streams, even as relatively minor contributors to the terrestrial flux of extraterrestrial material, is important in understanding the orbital evolution of meteoroid bodies, the rarity of potential asteroidal sources for ordinary chondrites, which account for more than $60 \%$ of meteorite falls (Greenberg and Nolan 1989), and apparent differences between meteorites currently falling and those that fell over the last few hundred thousand years and have been stored in the ice sheet of Antarctica (Koeberl and Cassidy 1991, Benoit and Sears 1993a, Wolf and Lipschutz 1995).

Precise orbits are known for only 4 meteorites (Wetherill and Chapman 1988. Brown et al. 1994). Orbital parameters have been estimated for another 40 meteorites from eyewitness accounts of their fall (Simonenko 1975, Wetherill and Chapman 1988), but these are of low accuracy. Limited orbital information can also be obtained from the local time of fall (Wetherill 1968). Meteorites with perihelia $\sim 1$ astronomical unit (AU) should usually strike the trailing side of the Earth. or the local PM, while meteorites with perihelia $<1 \mathrm{AU}$ should be more evenly spread over the leading and trailing sides. The large abundance of ordinary chondrites falling in the local PM relative to AM is interpreted as indicating that most of these meteorites had perihelia of $\sim 1 \mathrm{AU}$.

In this study we use the natural thermoluminescence of individual meteorites to estimate their closest approach to the Sun. Natural thermoluminescence is energy stored in crystals of certain minerals by ionizing radiation, such as high-energy galactic cosmic rays (Aitken 1985, McKeever 1985). This energy can be released in the form of visible light by heating.

The natural thermoluminescence (TL) of meteorites reflects both their thermal and irradiation history (McKeever 1980. Benoit et al. 1991). Virtually all ordinary chondrites have been exposed to galactic cosmic radiation for millions of years (Crabb and Schultz 1981), far longer than the $\sim 10^{5}$ years required to obtain TL equilibrium (Benoit $e t$ al. 1991). Depth effects on TL levels are minimal. TL profiles generally exhibiting $<30 \%$ variation in even the largest meteorites (Benoit and Chen 1996). The intense 
heating of atmospheric passage drains natural TL in the outermost few millimeters of meteorites (Sears 1975. Benoit and Chen 1996), but we have deliberately avoided sampling these regions in this study. Therefore, the natural TL of modern falls is controlled largely by the maximum tomperature experienced in space. which occurs at perihelion. The only exceptions are very large meteoroid bodies and meteoroids that have experienced shock heating within the last $10^{\circ}$ years, and both these exceptions appear to be very rare among the ordinary chondrites.

Following the treatment of Garlick and Gibson (1948), natural TL can be considered as the filling of "traps" by electrons put into the conduction band as a result of interaction with energetic particles. such as high-energy cosmic rays. The exact nature of these "traps" is presently unknown. but is generally considered to involve crystallographic defects and impurities (Prescott and Fox 1993). In crystals. populations of these "traps" are the source of TL. the electrons being freed from the traps and emitting a photon of light as the sample is heated. A typical TL experiment resulting in a "glow curve" involves heating a sample up to about $500^{\circ} \mathrm{C}$ in an inert atmosphere at a constant rate. while monitoring the amount of light given off by the sample as a function of temperature. In the present study we rearrange the basic equilibrium TL equation to solve for temperature (McKeever 1980. Benoit et al. 1991),

$$
T=\frac{-E}{k \ln \left[\frac{([N / n]-1) \ln (2) r}{s R}\right]}
$$

where $n / N$ is the measured TL level relative to saturation. $R$ is the radiation dose required to fill half the TL "traps." $k$ is Boltzmann's constant. and $r$ is the radiation dose rate. We use a dose rate of $0.01 \mathrm{~Gy} /$ year ( $1 \mathrm{rad} / \mathrm{year}$ ) in our calculations: the actual dose rate experienced by samples will depend on the depths of the samples in the meteoroid. a factor we discuss below. $E$ and $s$ are characteristic of the TL phosphors. sodic feldspar in the case of ordinary chondrites. at a particular portion of the glow curve and they are essentially constants for ordinary chondrites (McKeever 1980). We can then calculate the distance from the Sun $(X)$ from the calculated temperature assuming blackbody behavior.

$$
X=\left[\frac{0.25 \alpha H}{\varepsilon \sigma T^{4}}\right]^{1 / 2}
$$

where $\alpha$ and $\varepsilon$ are the absorptivity and emissivity of the surface of the meteoroid body, $H$ is the solar constant. and $\sigma$ is the Stefan-Boltzmann constant (Melcher 1981). Using

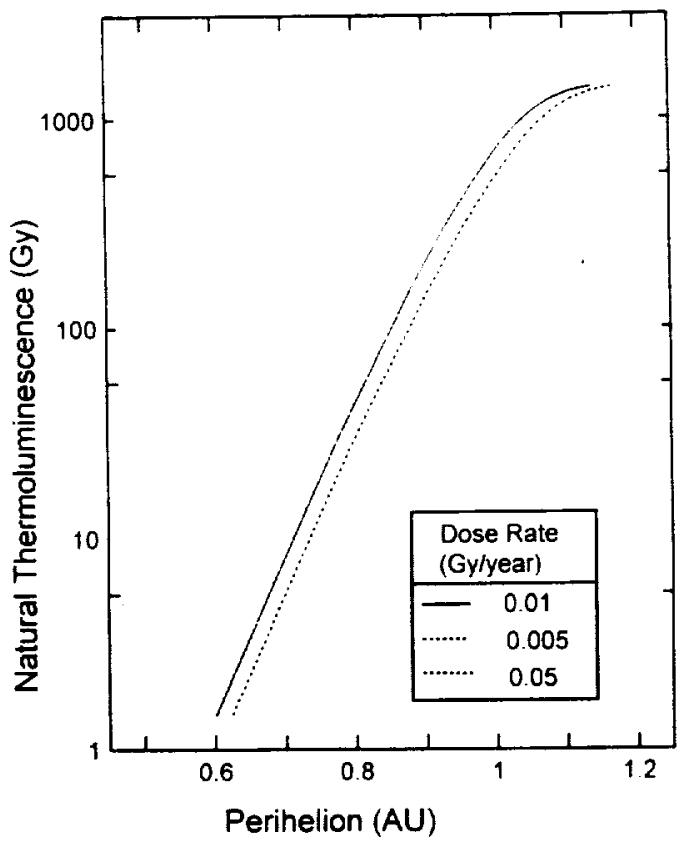

FIG. 1. Equilibrium natural thermoluminescence of ordinary chondrites at $250^{\circ} \mathrm{C}$ in the glow curve as a function of closest approach to the Sun (perihelion). Perihelion is given in astronomic units (AU).

a blackbody approximation in this case is appropriate because no asteroids, including those observed closely by space probes (Helfenstein et al. 1994). have atmospheres and the abundances of radioactive elements are too low in ordinary chondrites for these bodies to have any significant degree of internal heating during the last few million years. As a simplifying assumption, we set $\varepsilon$ to unity and estimate a value of $\alpha$ of 0.85 from the albedos of meteorite samples in the laboratory and remote-sensing measurements of asteroid surfaces (Chapman and Salisbury 1973. Helfenstein et al. 1994). In Fig. 1 we show the relationship between natural TL level and perihelia for ordinary chondrites derived from Eqs. (1) and (2). This calculation shows that equilibrium natural TL levels exhibit a strong dependence on perihelia for perihelia $<1.1 \mathrm{AU}$. We show curves for three dose rates, ranging from 0.005 to $0.05 \mathrm{~Gy} /$ year. In the following discussion we quote perihelion values using the 0.01-Gy/year curve, an apparently typical value for ordinary chondrite meteoroid bodies (Benoit and Chen 1996. and references therein). The 0.005- and 0.05-Gy/year curves are more appropriate for samples from very small and very large meteoroid bodies. respectively. and it is apparent that differences in meteoroid size have fairly minimal effect on our interpretation.

When we give a perihelion value for a particular meteorite. numerous uncertainties are included. some of which cannot be estimated with accuracy. Equation (2) is suscep- 
tible to uncertainties in albedo. Meteorite samples do exhibit some range of albedoes (Chapman and Salisbury 1973) and the relationship between the albedoes of meteorites and those of the surface of meteoroid bodies. of prime importance in this study, is not known. The major TL parameters. $E$ and $s$. appear to exhibit little or no significant variation among ordinary chondrites (e.g., McKeever 1980). but. as mentioned above. some uncertainty is imposed because the size of the meteoroid body before atmospheric passage and the position of the TL sample in the body are generally not known. resulting in some uncertainty in dose rate. Because these uncertainties cannot be quantified. we base most of our analysis on our original TL data. The quotation of perihelion value, either below or in our figures. should be regarded as an interpretation of these data.

\section{EXPERIMENTAL}

For each meteorite we crushed an approximately 250$\mathrm{mg}$ chip to a fine powder and removed metal (which is nonluminescent) with a hand magnet. We placed 4-mg aliquots of powder in copper pans which were then heated in a nitrogen atmosphere at a rate of $7.5^{\circ} \mathrm{C} / \mathrm{sec}$ from room temperature to $500^{\circ} \mathrm{C}$ in a Daybreak Nuclear and Medical Systems TL apparatus fitted with blue bandpass and IR filters (Corning 7-59 and 4-69). Three aliquots of each sample were analyzed and the results averaged. Induced TL was measured by the same procedure $5 \mathrm{~min}$ after exposure to a $20-\mathrm{Gy}$ dose from a ${ }^{90} \mathrm{Sr}-{ }^{91} \mathrm{Y}$ source. We used the ratio of the low-temperature $\left(250^{\circ} \mathrm{C}\right.$ in the glow curve) peak to the high-temperature $\left(400^{\circ} \mathrm{C}\right)$ peak to find the equivalent dose at $250^{\circ} \mathrm{C}$ in the glow curve, using a previously determined laboratory conversion equation (Hasan et al. 1987). The equivalent dose is the amount of radiation exposure needed to duplicate the observed natural TL level at a given temperature in the TL glow curve. A sample of a laboratory standard (the Dhajala H3.8 meteorite) was analyzed at the start and end of each measurement period as both a normalization standard and a check on the longterm stability of the apparatus.

Uncertainties in the natural TL data from analytical sources. as determined by triplicate measurements on each sample, are quite small. being generally a few percent of the natural TL level. except for TL levels below about 15 Gy that may have analytical uncertainties of up to $10-15 \%$. We did not collect multiple samples from each meteorite and therefore we cannot directly assess the uncertainties caused by depth dependence; however, detailed TL studies of other meteorites indicate that typical sample-to-sample heterogeneity in meteorites introduces uncertainties of no more than 10\% (Benoit and Chen 1996). With the exception of meteorites with very low natural TL levels $(<15$

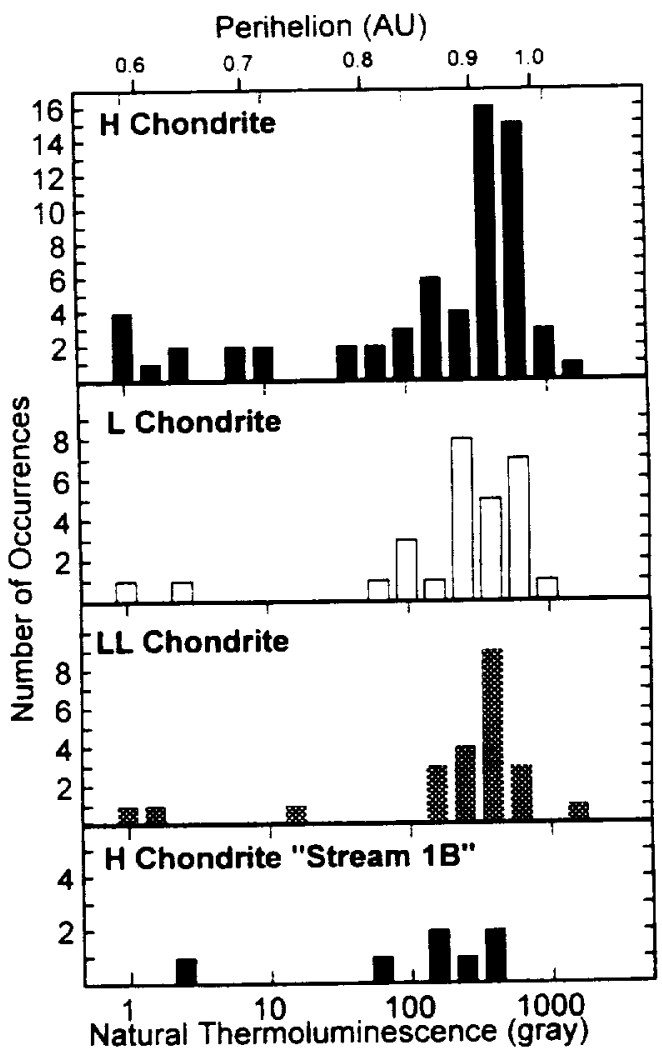

FIG. 2. Natural thermoluminescence at $250^{\circ} \mathrm{C}$ in the glow curve for ordinary chondrites. The distributions for $\mathrm{H}, \mathrm{L}$, and $\mathrm{LL}$ chondrites are fairly similar and correspond to orbits with perihelia between -0.75 and $\sim$ 1.0 AU. Members of a possible $\mathrm{H}$ chondrite orbital debris stream (Dodd et al. 1993) have a distribution similar to that of other ordinary chondrites.

Gy), uncertainties in our data are no larger than the size of width of the boxes on our histograms (Fig. 2).

\section{RESULTS}

We have determined natural TL levels for 118 equilibrated ordinary chondrites (Fig. 2). Our previous work indicates that the natural TL levels of equilibrated ordinary chondrites are not affected by nonthermal losses or anomalous fading (Wintle 1973. Sears et al. 1991). There are no significant differences in natural TL between the major classes. the H. L. and $L L$ chondrites. all classes having natural TL values of 50 to $1000 \mathrm{~Gy}$, with a few outliers at lower values.

\section{DISCUSSION}

We can convert the natural TL data for individual meteorites to perihelion using Eqs. (1) and (2). As noted above. such a conversion carries uncertainties that cannot be quantified. but differences of a few hundredths of an $\mathrm{AU}$ 


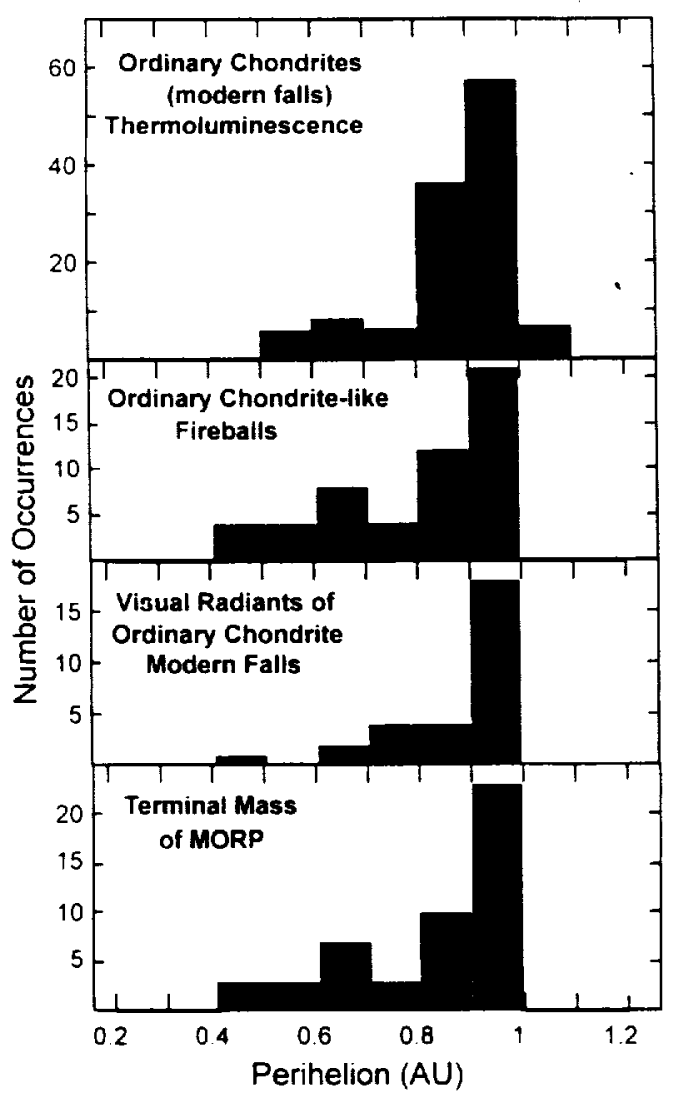

FIG. 3. An interpretation of the natural TL data for ordinary chondrites (Fig. 2), using Eqs. (1) and (2) to convert the TL data to perihelia. For comparison. perihelion distributions derived from chondrite-like lircballs (Wetherill and ReVelle 1981). visual radiants of observed meteorite falls (Simonenko [475). and MORP fireballs (Halliday el al. 1983) are also shown. In general. the distribution derived from TL is similar to other distributions. and is most similar to those exhibited by chondritelike tiretralls and the distribution derived from MORP fireballs.

are probably signiticant in comparisons between meteorites. The range of natural TL levels corresponds to perihelia of 0.75 to $1.0 \mathrm{AU}$. with an average around $0.95 \mathrm{AU}$. with just a few outliers with perihelia $<0.75$ and $>1.0 \mathrm{AU}$ (Fig. 2). This distribution is in general agreement with the distribution suggested by observed meteorite falls and also with the more numerous meteor and fireball radiant data (Fig. 3) (Wetherill and Chapman 1988). The distribution derived from the natural TL of meteorites is most similar to the distribution observed for fireballs attributed to ordinary chondrite objects. defined by two different methods (Wetherill and ReVelle 1981. Halliday et al. 1983), and differs from the distribution derived from visual radiants of ordinary chondrite falls by having a larger proportion of meteorites with perihelia between 0.8 and $0.9 \mathrm{AU}$. The most likely cause of this difference. as discussed by Wetherill and ReVelle (1981), is biases inherent in the estimates of orbits from radiants observed by untrained persons.
The outliers with natural TL levels $<50$ Gy (Fig. 2) are not discussed further here and are not considered in our statistics. because it is possible that some of these meteorites had their TL levels lowered by recent shock heating or we mav have accidently sampled material near the fusion crust created during atmospheric passage. Thus. for these meteorites. the natural TL level could reflect a heating event other than closest approach to the Sun.

As was noted above. one of the key observations that led to the delineation of the orbits and source of meteorites was the AM/PM time-of-fall distribution (Wetherill 1968). We would expect such differences also to be reflected in the TL data and we do find such differences in at least two of the major ordinary chondrite classes (Fig. 4). The $\mathrm{H}$ chondrites that fell in the local AM have a mean natural TL level similar to that of chondrites that fell in the PM

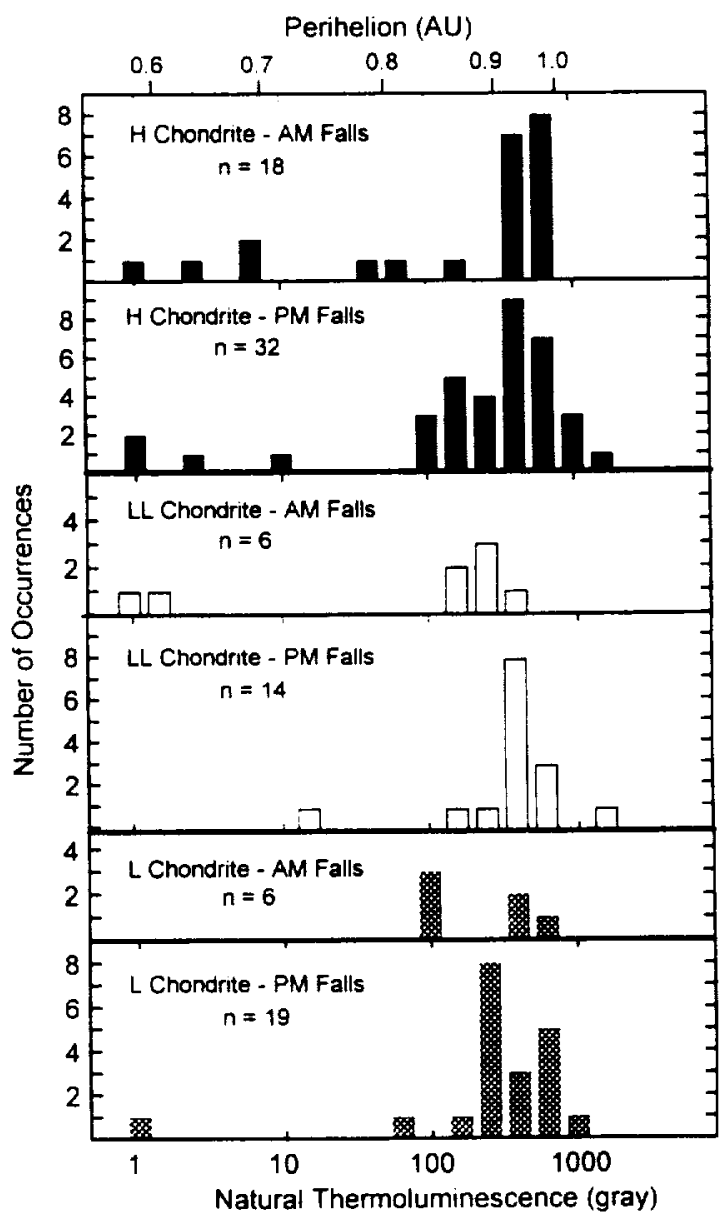

FIG. 4. Natural thermoluminescence distributions for $H$. L. and LL chondrites that fell in the AM and Ps. local time. For $\mathrm{H}$ chondrites the iM falls exhibit a lower degree of variation than $P M$ falls. whereas for LL chondrites the AM falls generally have lower TL levels than PM falls Only meteorites with natural TL levels $>50$ Gy are considered in our statistics and the total number used for each class and time-of-fall is given by $n$. 
(about $300 \mathrm{~Gy}$ ), but the AM falls had a much smaller standard deviation. lacking the large number of samples with natural TL of $100-300 \mathrm{~Gy}$ present in the PM falls (Fig. 2). The $F$ test. used to examine equality of variance between data sets (Davis 1986). indicates that this difference is significant at the $95 \%$ confidence level and is thus probably not simply due to the smaller number of $A M$ falls in our study. The LL chondrites that fell in the local $A M$ have lower natural TL levels than PM falls, the difference being significant at the $99 \%$ confidence level according to the $t$ test, but the distributions have similar variances. There are no significant differences in the TL distributions of $L$ chondrite $A M$ and PM falls.

Our interpretation of these data is that the AM falls of LL chondrites have perihelia between 0.85 and $0.90 \mathrm{AU}$. whereas the PM falls are dominated by meteorites with perihelia between 0.85 and $1.0 \mathrm{AU}$. On the basis of the orbital dynamics arguments of Wetherill (1968), it can be argued that these data support the conclusion that $\mathrm{LL}$ chondrites are a steady-state population derived from the asteroid belt via orbital resonances that preferentially place them in orbits with perihelia of $\sim 1 \mathrm{AU}$. These bodies are short-lived. being removed from the inner solar system by interactions with planets on the $10^{6}$-year time scale. and only a few objects achieve orbits with perihelia significantly $<1$ AU. Our data indicate that very few LL chondrites that reach Earth have perihelia $<0.8 \mathrm{AU}$. An alternative interpretation of these data is that the $A M$ and $P M$ fall samples experienced different dose rates as a result of $A M$ falls being larger than PM falls. Our investigations of TL profiles in meteorites, however, suggest that the observed difference would require all AM falls to be larger than typical meteoroid bodies (Benoit and Chen 1996). Such a conclusion is not supported by the size-dependent ratios of cosmogenic noble gases in LL chondrites (Graf and Marti 1994).

The $H$ chondrites have a more complicated apparent orbital distribution (Fig. 4). While the AM falls have perihelia values of $\sim 0.95 \mathrm{AU}$, the PM falls. which should be dominated by meteorites with perihelia of about $1 \mathrm{AU}$. exhibit a range of 0.80 to $1.0 \mathrm{AU}$ with a significant number $<0.9 \mathrm{AU}$. One possible interpretation of this result is that the PM falls exhibit an extreme range of preatmospheric sizes and the AM falls are very homogeneous in size. This interpretation is not supported by the ratios of cosmogenic noble gases found in these meteorites such as ${ }^{21} \mathrm{Ne}$ and ${ }^{2} \mathrm{Ne}$. which vary as a function of meteoroid size (Eugster 1988. Michel et al. 1991).

An alternative interpretation of these data is that the $\mathrm{H}$ chondrites with TL levels between 100 and $300 \mathrm{~Gy}$ in the PM falls experienced shock heating within the last $10^{5}$ years, the time required for TL to rebuild to thermally controlled equibrium: however, these meteorites display no petrographic evidence for high levels of shock. Another possibil-
TABLE I

$H$ Chondrites in an Inferred Orbital Cluster

\begin{tabular}{|c|c|c|c|}
\hline Meteorite & Class & $\begin{array}{l}\text { Cosmic ray } \\
\text { cxposure } \\
\text { age (Ma) }\end{array}$ & $\because \mathrm{Ne} / 2 ! \mathrm{Ne}$ \\
\hline Barbotan & H5 & 9 & 1.10 \\
\hline Beaver Creek & $\mathrm{H} 4$ & 29 & 1.04 \\
\hline Charsonville & H6 & 55 & 1.06 \\
\hline Forest City & $\mathrm{H} 5$ & 6 & 1.08 \\
\hline Gopalpur & $\mathrm{H} 5$ & 10 & 1.24 \\
\hline Lillaverke & H5 & - & - \\
\hline Noblesville & $\mathrm{H} 4$ & - & - \\
\hline Pokhra & $\mathrm{H} 5$ & 3 & 1.24 \\
\hline Pribram & H5 & 15 & 1.11 \\
\hline Rochester & H6 & 25 & 1.07 \\
\hline St. Germain du Pinel & H6 & 36 & 1.14 \\
\hline Zhovtnevy & H5 & 42 & 1.08 \\
\hline
\end{tabular}

ity is that these meteorites were in orbits with perihelia of about $0.8 \mathrm{Au}$ but moved to orbits with perihelia of $\sim 1 \mathrm{AU}$ within the last $10^{5}$ years. Such rapid orbit changes are possible, or even expected, for Earth-crossing asteroids as a result of orbital interactions with planets (Galibina and Terent'eva 1987, Farinella et al. 1993). We have previously described a large Antarctic meteorite shower that apparently underwent rapid evolution from orbits with perihelia >1.2 AU to $~ 1 \mathrm{AU}$ (Benoit and Sears 1993b). This interpretation, however, requires that these meteorites changed orbit as a group. This could occur if these meteorites were part a single body that fragmented after changing orbit, but this seems unlikely due to their petrographic diversity (encompassing petrographic types 4,5 , and 6) and wide range of cosmic ray exposure ages (Table I). Alternatively, they could have formed an orbital debris "stream." similar to those that produce meteor showers (Olsson-Steel 1988. Halliday et al. 1990). In this case. these meteorites reach Earth only when Earth's orbit intersects the stream.

Dodd et al (1993) have examined the time-of-fall statistics for $\mathrm{H}$ chondrites in an attempt to identify possible orbital streams among historical falls. They proposed that a small subset of $\mathrm{H}$ chondrites (including some of the PM falls with apparent perihelia of $\sim 0.8 \mathrm{AU}$ in our data set) were in an orbital stream. called $\mathrm{H}$ Cluster 1 . Our analysis of 7 of 11 potential members of this stream, however, indicates that there is no difference between their TL distribution and that of other $\mathrm{H}$ chondrites (Fig. 2), suggesting that these meteorites did not have similar perihelia in their recent history and thus were probably not members of a modern orbital stream. This conclusion. however, rests on the assumption that a meteoroid stream has a stable orbit on the time scale reflected in the collection of modern falls. namely, on the scale of a few decades up to about a century, and thus that such streams are similar to the debris streams 


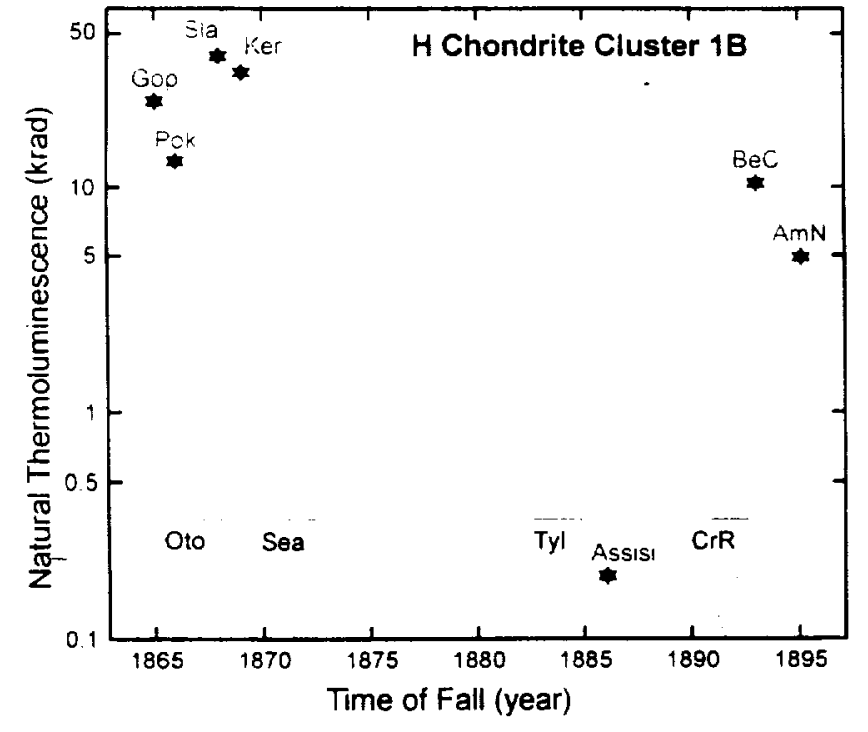

FIG. 5. Natural thermoluminescence levels of $\mathrm{H}$ chondrites from the proposed Cluster IB orbital stream of Dodd et al. (1993). plotted as a function of their year of fall. Meteorites in triangles along the bottom axis were not analyzed in this study. Meteorites that fell before about the year 1870 exhibit a fairly tight range of natural TL levels. consistent with perihelia slightly less than $0.95 \mathrm{AU}$. Those that fell after the year 1885 have lower natural TL levels. possibly reflecting perihelia less than about 0.85 AU. AmN. Ambapur Nagla: BeC. Beaver Creek: CrR. Cross Roads: Gop. Gopalpur: Ker. Kernouvé; Oto. Otomi: Pok, Pokra: Sea. Searsmont: Sla. Slavetic: Tyl, Tysnes Island.

responsible for meteor showers. Dodd et al. (1993) point out that a subset of the proposed $\mathrm{H}$ Chondrite Cluster 1 , the $1 \mathrm{~B}$ stream, is not so well behaved and, although the members of the proposed stream fell over a period of about 30 years $(1865-1895)$, there is a pronounced gap within this range of about 12 years (1872-1883) in which no potential members fell. They suggest this reflects the narrow width of the stream and perhaps an orbit inclined to the ecliptic. We note that there is an apparent difference in the TL levels of meteorites from the proposed stream depending on their year of fall (Fig. 5). Meteorites that fell before the gap appear to have moderate natural TL levels. consistent with perihelia of slightly less than $0.95 \mathrm{AU}$. Meteorites that fell after the gap, however, have significantly lower natural TL levels. consistent with perihelia of $<0.85 \mathrm{AU}$, and one. Assisi. may have had a perihelion of about 0.6 AU (Benoit et al. 1991). It is unlikely that Assisi experienced TL loss due to a recent shock event. having a low petrographic shock level (Dodd et al. 1993) and a TL sensitivity of $4.3 \pm 0.2$ relative to the Dhajala meteorite, similar to other unshocked equilibrated ordinary chondrites (Haq et al. 1988). The apparent gap in the proposed Cluster 1B could reflect a change in the orbit of the stream, with the members of the stream achieving much lower perihelia after the gap. or. conversely. this could reflect the sampling of different portions of a narrow stream by slight variations in the Earth's orbit. From the perspective of Fig. 4. in contradiction of the summary data of Fig. 2, it appears more likely that the proposed Cluster $1 \mathrm{~B}$ is actually a meteoroid stream. albeit a rapidly evolving one, with the stream breaking up in the second encounter. We stress, however. that this conclusion is of necessity based on a very small number of meteorites.

\section{CONCLUSIONS}

Only a small fraction $(<25 \%)$ of the $\mathrm{H}$ chondrites we have analyzed are involved in an inferred orbital cluster. In general. our data indicate that the remainder fit into the model of meteorid orbital distribution suggested by Wetherill (1968). Most ordinary chondrites appear to have been in orbits with perihelia between 0.8 and $1.0 \mathrm{AU}$ prior to reaching Earth, and therefore they were probably derived as individuals from the asteroid belt through orbital resonance with the major planets. We find differences between the orbital distributions of meteorites falling in the local morning and those falling in the afternoon and evening. As predicted from indirect data, most ordinary chondrites were in orbits with perihelia $>0.85 \mathrm{AU}$, but we suggest that a small fraction of meteorites have experienced recent histories involving rapid orbital changes. We find some support for the existence of a rapidly evolving stream of $\mathrm{H}$ chondrites. previously suggested on the basis of time-of-fall data. In general, it can be argued that minor. but perhaps important, changes have occurred in the numbers and types of meteorites reaching Earth on the $<10^{5}$. year time scale.

\section{ACKNOWLEDGMENTS}

We thank Dr. Stanley G. Love and an anonymous reviewer for their comments on this manuscript. We also thank the museums and organizations that contributed samples to this study, namely, the Center for Meteorite Studies. Arizona State University; the British Museum; the Canadian Geological Survey: the Field Museum of Natural History. Chicago; the University of California, Los Angeles: the Institute of Meteoritics. University of New Mexico; the U.S. Natural History Museum: and the Chinese Academy of Sciences. We also thank M. E. Lipschutz and S. F. Wolf (Purdue University) for samples and discussions. This study funded by the National Science Foundation and the National Aereonautics and Space Agency of the United States.

\section{REFERENCES}

AITKEN. M. J. 1985. Thermoluminescence Dating. Academic Press. London.

BENOIT, P. H., AND Y. CHEN 1996. Galactic cosmic-ray-produced thermoluminescence profiles in meteorites, lunar samples and a terrestrial analog. Radiat. Meas. 26, 281-289.

BenOIT. P. H., AND D. W. G. Sears 1993a. Breakup and structure of an $\mathrm{H}$-chondrite parent body: The $\mathrm{H}$-chrondite flux over the last million years. Icarus 101, 188-200. 
BENOtT. P. H.. AND D. W. G. SeARS 1993b. A recent meteorite shower in Antarctica with an unusual orbital history. Earth Planet. Sci. Lett. 120, 463-471

Benolt, P. H., D. W. G. Sears, and S. W. S. Mckeever 1991. The natural thermoluminescence of meteorites: II. Meteorite orbits and orbital evolution. lcarus 94, 311-325.

Binzel. R. P., S. Xu, S. J. Bus, M. F. Skrutskie. M. R. Meyer. P. KNEZEK. AND E. S. BARKER 1993. Discovery of a main-belt asteroid resembling ordinary chondrite meteorites. Science 262, 1541-1543.

Brown. P. Z. Ceplecha. R. L. Hawkes. G. Wetherill. M. Beech. ANd K. Mossman 1994. The orbit and atmospheric trajectory of the Peekskill meteorite from video records. Nature 367, 624-626.

Chapman, C. R.. AND J. W. Salisbury 1973. Comparisons of meteorite and asteroid spectral reflectivities. Icarus 19, 507-522

CRABb, J., AND L. SChultz 1981. Cosmic-ray exposure ages of the ordinary chondrites and their significance for parent body stratigraphy. Geochim. Cosmochim. Acta 45. 2151-2160.

DAvis. J. C. 1986. Statistics and Data Analvsis in Geology, 2nd ed. Wiley, New York.

Dodd, R. T., S. F. Wolf, and M. E. Lipschutz 1993. An $\mathrm{H}$ chondrite stream: Identification and confirmation. J. Geophys. Res. 98, 15105 15118.

Drummond. J. D. 1991. Theoretical meteor radiants for Apollo. Amor and Aten asteroids. Icarus 49, 143-153.

Eligster O. 1988. Cosmic-ray production rates for ${ }^{3} \mathrm{He},{ }^{21} \mathrm{Ne} .{ }^{38} \mathrm{Ar} .{ }^{83} \mathrm{Kr}$. and ${ }^{126} \mathrm{Xe}$ in chondrites based on $81 \mathrm{Kr}-\mathrm{Kr}$ exposure ages. Geochim. Cosmochim. Acra 52, 1649-1662.

Farinella, P., C. Froeschlé. and R. Gonczi 1993. Meteorites from the asteroid 6 Hebe. Cel. Mech. Dyn. Astron. 56, 287-305.

Galibina, I. V., and A. K. Terent'eva 1987. The Innisfree meteorite: Dynamic history of the orbit: A possible family of meteoric bodies Solar Syst. Res. 21, 160-166.

Garlick, G. F. J., AND A. F. Gibson 1948. The electron trap mechanism of luminescence in sulphide and silicate phosphors. Proc. Phys. Soc. Sect. A 60, 574-590.

Graf, Th., AND K. Marti 1994. Collisional records in LL-chondrites. Meteorifics 29, 643-648.

Greenberg. R., and M. C. Nolan 1989. Delivery of asteroids and meteorites to the inner Solar System. In Asteroids II (R. P. Binzel. T. Gehrels, and M. S. Matthews. Eds.), pp. 778-804. Univ. of Arizona Press. Tucson.

HALLIDAY. I. 1987. Detection of a meteorite "stream": Observations of a second meteorite fall from the orbit of the Innisfree chondrite. Icarus 69. $550-556$

halliday, I., A. A. Griffen, and A. T. Blackwell 1983. Meteorite orbits from observations by camera networks. In Highlights of Astronomy (J. Klinger. D. Benest, A. Dollfus, and R. Smoluchowski. Eds.), Vol. 6. pp. 399-404. Reidel, Dordrecht.

Halliday, I.. A. T. Blackwell, AND A. A. GRiffin 1990. Evidence for the existence of groups of meteorite-producing asteroidal fragments Meteoritics 25, 93-99.
Haq, M.. F. A. Hasan, and D. W. G. Sears 1988. Thermoluminescence and the shock and reheating history of meteorites: IV. The induced TL properties of type 4-6 ordinary chondrites. Geochim. Cosmochim. Acta 52, 1679-1689.

Hasan, F. A.. M. HaO, and D. W. G. Sears 1987. The natural thermoluminescence of meteorites: I. Twenty-three Antarctic meteorites of known ${ }^{26}$ Al content. Proc. Lunar Planet. Sci. Conf., 17th. J. Geophys. Res. 92, E703-E709.

Helfenstein. P.. AND 23 colleagues 1994 . Galileo photometry of asteroid 951 Gaspra. Icarus 107, 37-60.

Koeberl. C., and W. A. Cassidy 1991. Differences between Antarctic and non-Antarctic meteorites: An assessment. Geochim. Cosmochim. Acta 55, 3-18.

McKeever, S. W. S. 1980. The analysis of thermoluminescence glowcurves from meteorites. Mod. Geol. 7, 105-114.

McKeever, S. W. S. 1985. Thermoluminescence of Solids. Cambridge Univ. Press, Cambridge, England.

MELCHER. C. L. 1981. Thermoluminescence of meteorites and their orbits. Earth Planet. Sci. Lett. 52, 39-54.

Mrchel, R., P. Dragovitsch, P. Cloth, G. Dagge, and D. Filges 1991. On the production of cosmogenic nuclides in meteoroids by galactic protons. Meteoritics 26, 221-242.

Oberst, J.. AND Y. NaKamura 1991. A search for clustering among the meteoroid impacts detected by the Apollo lunar seismic network. Icarus 91, 315-325.

OLSSON-STEEL. D. 1988. Identification of meteoroid streams from Apollo asteroids in the Adelaide radar orbit surveys. Icarus 75, 64-96.

Prescott. J. R., AND P. J. Fox 1993. Three-dimensional thermoluminescence spectra of feldspars. J. Phys. D Appl. Phys. 26, 2245-2254.

SEARS, D. W. G. 1975. Thermoluminescence studies and the preatmospheric shape of Estacado meteorite. Earth Planet. Sci. Lett. 26, 97-104.

Sears, D. W. G., P. H. Benott, H. Sears, J. D. Batchelor, and S. SYMEs 1991. The natural thermoluminescence of meteorites: III. Lunar and basaltic meteorites. Geochim. Cosmochim. Acta 55, 3167-3180.

Simonenko, A. N. 1975. Orbital Elements of 45 Meteorites. Atlas, Nauka, Moscow.

WETHERILL. G. W. 1968. Stone meteorites: Time of fall and origin. Science 159, 79-82.

Wetherill, G. W, 1985. Asteroidal source of ordinary chondrites. Meteoritics $20,1-22$.

WETHERILL, G. W. AND C. R. ChAPMAN 1988. Asteroids and meteorites In Meteorites and the Early Solar System (J. F. Kerridge and M. S. Matthews. Eds.). pp. 35-67. Univ. of Arizona Press. Tucson.

WetheriLl, G. W.. AND D. O. ReVELLE 1981. Which fireballs are meteorites? A study of the Prairie Network photographic meteor data. Icarus 48, 308-328.

WINTLE, A. 1973. Anomalous fading of thermoluminescence in mineral samples. Nature 245, 143-144.

WOLF. S. F., AND M. E. LipsChUTZ 1995. Chemical studies of $H$ chondrites: 6. Antarctic/non-Antarctic compositional differences revisited. J. Geophys. Res. 100, 3335-3349. 


\section{-}

